\title{
Head and Neck Cancer: Closer Look at Patients Quality of Life
}

\author{
Azza N. Taher \\ Department of Radiotherapy, National Cancer Institute, Cairo University, Cairo, Egypt \\ Email: ataher3@yahoo.com
}

Received 21 December 2015; accepted 26 February 2016; published 29 February 2016

Copyright (C) 2016 by author and Scientific Research Publishing Inc.

This work is licensed under the Creative Commons Attribution International License (CC BY). http://creativecommons.org/licenses/by/4.0/

(c) (i) Open Access

\begin{abstract}
Head and neck cancer (HNC) as a disease and its treatment have a disproportionate impact on all aspects of patients' quality of life (QOL). QOL is a multidimensional construct of an individual's subjective assessment of the impact of an illness or treatment on his/her physical, psychological, social and somatic functioning and general well-being [1]. Thus, QOL has become an important treatment outcome in HNC [2]. Objective: It is to evaluate the quality of life of patients dealing with squamous cell carcinoma of the head and neck treated in the radiotherapy department, National Cancer Institute, Cairo University. Material and methods: Starting from March 2012 to December 2013, 87 patients with histopathologically proven Head and Neck Squamous Cell Carcinoma (HNSCC) were taking part in our prospective study. The patients' QOL answers to the questionnaire were collected for both the EORTC QLQ-C30 and the EORTC Head and Neck questionnaires (QLQ-H\&N35) and then all points were transformed to a 0 - 100 scale according to the guidelines of the EORTC. Certain clinical factors were chosen to evaluate their effect on the QOL; site of primary tumor, clinical stage, and mode of therapy applied: radiotherapy versus chemo-radiotherapy (CRT). Moreover, age, gender and smoking were studied. Results: Majority of the items assessed in the QLQ-C30 and QLQ-H\&N35 questionnaire showed that they were negatively impacted at the end of treatment. As for the factors that had a negative effect on the QOL, they comprised; female gender $(p<0.05)$, age $<60$ years $(p<0.05)$, smoking $(p<0.01)$, clinical stage III and IV $(p<0.05)$, larynx and hypopharynx as a site of primary $(p<0.01)$, and CRT $(p<0.01)$. Conclusions: Results of our study showed that treatment options had a significant negative effect on the QOL by the end of the treatment course. Moreover, our results showed that age, gender, smoking habit, tumor site, and clinical stage of disease had a significant impact on the QOL of HNSCC patients.
\end{abstract}

\section{Keywords}

Head and Neck Cancer, Radiotherapy, Chemo-Radiation, Quality of Life 


\section{Introduction}

Patients with head and neck cancer experience significant changes in their quality of life (QOL) associated with the disease and treatment adverse effects. Common problems include difficulty with speech, respiration, and eating, as well as the psychological impact of loss of function and physical disfigurement. With the advent of more aggressive treatment modalities, QOL assessment has become an essential part of clinical care and research studies.

QOL is a term that is used to describe the various changes and effects that the disease imposes on the patient's different aspects of life as well as the side effects of the treatment in a wider scope than the usual methods of describing side effects of treatment. Notably, QOL is a subjective tool that gives information about the various symptoms from the patient's point of view [3]. Therefore, the effects inflicted upon the QOL can greatly depend on the treatment modality and the patient's method of reacting to certain situations [4].

The European Organization for Research of Life Questionnaire Core 30 Items (EORTC QLQ-C30) including the H\&N-35 module are the most used and tested QOL tool in the field of oncology [5] [6].

The standard of care for HNSCC patients pertains the use of combined treatment modalities, though more effective yet with higher rates of side effects, a closer look at the patients' wellbeing, expectations, and the personal perception of the treatment's effects is now a necessity. The choice of the treatment modality calls for weighing and considering various factors and options by the multidisciplinary treating team as well as the patient which is a difficult and complex task. Thus the evaluation of QOL supplies more information that aids in comprehending the difference between the various treatment modalities as regards their impact on the physical, psychological, social and functional aspects of the patients [3].

The aim of our study is to evaluate the quality of life of patients with squamous cell carcinoma in the head and neck, undergoing treatment. As well as, the effect of certain clinical and social factors on the QOL is evaluated too.

\section{Material and Methods}

A prospective clinical study that enrolled 87 patients with HNSCC that presented to the Radiation therapy department of the National Cancer Institute, Cairo University, it started from March 2012 and ended in December 2013. The patients had received RT or chemo-radiotherapy (CRT) treatment with or without surgical resection. The inclusion criteria were: Histopathologically confirmed squamous cell carcinoma of head and neck cancer (HNSCC) with any of the following subsites: oropharynx, hypopharynx, larynx, oral cavity, no evidence of distant metastases, performance status ECOG (0-1), the prescribed radiation dose fraction was 2 Gy with a maximum total dose of $70 \mathrm{~Gy}$ in case of radical radiotherapy. Clinical factors such as the site of the tumor, clinical stage, treatment modality (RT, CRT), and social factors (age, gender, smoking habit) were evaluated. The patients' characteristics are presented in Table 1. The standardized EORTC-QLQ-C30 (version 3.0) questionnaire and QLQ-H\&N35 module were used for the evaluation of QOL. The items covered in QLQ-C30 questionnaire are focused on evaluating general health, physical, emotional and social aspects. It comprises 30 questions grouped into the 5 functional scales. The questionnaire also includes 3 symptomatic scales-fatigue, nausea and pain-as well as 6 single questions evaluating the intensity of the following symptoms: dyspnea, sleeplessness, lack of appetite, constipation, diarrhea and financial problems. The last two questions deal with the overall health assessment. There is a four-degree scale in the answers to the questions in the questionnaire (never 1 , sometimes 2, often 3, very often 4). The QLQ-H\&N35 questionnaire deals with specific symptoms related to tumors of the head and neck, and effects of treatment. It has 35 questions grouped into 7 scales: pain, swallowing, senses, speaking, eating in the company of others, social contacts, sexuality, and 11 individual questions concerning teeth problems, difficulties with opening the mouth, oral cavity dryness, the presence of thick saliva, coughing, illness awareness, taking pain killers, using food supplements, and losing or gaining weight. Similarly, to the core questionnaire, a patient gave one answer to each question, and the answers had a four-degree scale.

\section{Statistical Analysis}

The results of both questionnaires were extrapolated according to the ESTRO guidelines; where the raw coefficient was calculated and linear transformation was done. A score ranging between 0 and 100 for all the scales was thus created. In the case of the QLQ-C30 questionnaire a higher score corresponds to a better level for the 
Table 1. Patients sociodemographic characteristics and treatment type.

\begin{tabular}{|c|c|c|}
\hline Variables & Number of patients & Percentage \\
\hline Gender: Male & 72 & 82.80 \\
\hline Female & 15 & 17.20 \\
\hline Age (years): $\leq 50$ & 12 & 13.8 \\
\hline $51-60$ & 39 & 44.8 \\
\hline$>60$ & 36 & 41.4 \\
\hline Smoking: yes & 55 & 63.3 \\
\hline No & 32 & 36.7 \\
\hline \multicolumn{3}{|l|}{ Tumor site: } \\
\hline Oral cavity \& oroph. & 39 & 45 \\
\hline Larynx \& hypopharynx & 48 & 55 \\
\hline \multicolumn{3}{|l|}{ Clinical Stage: } \\
\hline $\mathrm{I}+\mathrm{II}$ & 30 & 34.5 \\
\hline III + IV & 57 & 65.5 \\
\hline \multicolumn{3}{|l|}{ Treatment Type: } \\
\hline Surgery + RT & 12 & 18.5 \\
\hline Chemo-RT & 27 & 33.3 \\
\hline Surgery + chemo-RT & 3 & 3.7 \\
\hline RT alone & 36 & 44.5 \\
\hline Total & 87 & 100 \\
\hline
\end{tabular}

function, meaning a better QOL. As for the symptoms, a higher score denotes worsening of the symptom. As for the QLQ-H\&N35 questionnaire, a higher score denotes worsening of the problem; worse QOL. For the assessment of QOL before and at the end of treatment, Student's t-test was used for the statistical calculations. The statistical analysis and all the calculations were made with SPSS 17.0.

\section{Results}

Our present study included 87 patients their ages ranged between 43 and 74 years, with mean age of 57 years, between March 2012 and December 2013. Majority of the patients were males (82.8\%), majority were smokers (63.3\%). Tumor size was assessed according to the TNM classification [7], almost two thirds of the cases were T3 and T4 (65.5\%). As for the primary lesion it was more frequently located on the tongue or pharynx (22.7\% each), the larynx (18.2\%), lips (13.6\%) and palate \& maxilla (9\%). Almost half of the patients were submitted to radiotherapy (RT) only (44.5\%), followed by combined treatment with chemo and radiotherapy (CRT) (33.3\%) whereas surgery and radiotherapy as a modality was implemented in $18.5 \%$ of the patients, besides surgery, radiotherapy and chemotherapy combined (Table 1).

All of our patients answered both questionnaires EORTC QLQ-C30 and QLQ-H\&N35 before and at the end of treatment. For the EORTC QLQ-C30 questionnaire, a higher score for a function equals a better level of functioning meaning a better QOL. Upon interpretation a difference of 10 or more points on a scale from 0 to 100 was regarded as a clinically significant score improving or worsening the QOL. This is a common cut-off value used in studies of QOL, that was introduced by King and Osoba et al. [8] [9]. Whereas, a difference of 20 points indicates a high significance and a 5-point difference only denotes clinical importance. In the questionnaire QLQ-C30 nine of the thirty analyzed components showed statistically significant differences upon analysis and thus had a very negative impact on QOL (Table 2). The QLQ-H\&N35 specific questionnaire (Table 3) shows 7 parameters that worsened with great significance after end of treatment, namingly sense of taste and smell, pain, swallowing, dryness of mouth, thickened saliva, feeding problems and cough. Despite the fact that there was significantly positive effect of treatment on stopping weight loss yet the weight gain process didn't reach a significant level. Smoking worsened the QOL of patients at the end of treatment. Higher clinical stage 
Table 2. EORTC QLQ-C30 of 87 patients assessed at onset and end of treatment.

\begin{tabular}{|c|c|c|c|c|}
\hline \multirow{3}{*}{$\begin{array}{l}\text { Scale Items } \\
\text { Symptoms }\end{array}$} & \multicolumn{4}{|c|}{ Treatment Period } \\
\hline & Onset & Post & \multirow{2}{*}{ Diff. Bet. Means } & \multirow{2}{*}{ p-Value } \\
\hline & Mean (SD) & Mean (SD) & & \\
\hline \multicolumn{5}{|c|}{ Functions } \\
\hline Physical & 82.95 (14.88) & 77.10 (18.29) & 5.85 & $<0.01$ \\
\hline Emotional & $61.20(21.75)$ & $52.70(22.75)$ & 8.49 & 0.502 \\
\hline Cognitive & 84.93 (18.03) & $80.54(23.45)$ & 4.39 & $<0.01$ \\
\hline Social & 81.89 (23.33) & $73.20(29.32)$ & 8.69 & $<0.01$ \\
\hline Role Performance & 83.68 (19.09) & $75.23(27.04)$ & 8.45 & $<0.01$ \\
\hline \multicolumn{5}{|c|}{ Symptoms } \\
\hline Fatigue & 34.92 (22.29) & $50.21(27.06)$ & -15.28 & $<0.01$ \\
\hline Nausea \& Vomiting & $9.97(14.72)$ & $28.59(27.00)$ & -18.61 & $<0.01$ \\
\hline Pain & 26.88 (23.83) & $43.22(30.10)$ & -16.34 & $<0.01$ \\
\hline Dyspnea & $7.3(18.1)$ & $19.8(32.1)$ & -12.50 & 0.368 \\
\hline Insomnia & $34.80(30.38)$ & 39.7 (32.38) & -04.90 & $<0.05$ \\
\hline Loss of Appetite & 22.49 (27.07) & $61.84(26.32)$ & -39.35 & $<0.01$ \\
\hline Constipation & $2.0(8.00)$ & $11.8(28.7)$ & -09.80 & 0.180 \\
\hline Diarrhea & $1.9(8.10)$ & 9.8 (25.7) & -07.90 & 0.368 \\
\hline Financial Difficulty & $24.5(36.4)$ & $32.3(40.8)$ & -07.80 & 0.168 \\
\hline General Health Status/ QOL & $66.7(23.0)$ & $71.7(20.4)$ & -05.00 & 0.280 \\
\hline
\end{tabular}

Table 3. EORTC QLQ-H\&N35 of 87 patients assessed at onset and end of treatment.

\begin{tabular}{|c|c|c|c|c|}
\hline \multirow{3}{*}{$\begin{array}{l}\text { Scale Items } \\
\text { Symptoms }\end{array}$} & \multicolumn{4}{|c|}{ Treatment Period } \\
\hline & \multirow{2}{*}{\begin{tabular}{c|c|} 
Onset \\
Mean (SD)
\end{tabular}} & \multirow{2}{*}{$\begin{array}{c}\text { Post } \\
\text { Mean (SD) }\end{array}$} & \multirow{2}{*}{ Diff. Bet. Means } & \multirow{2}{*}{ p-Value } \\
\hline & & & & \\
\hline Pain & $20.85(21.18)$ & $50.59(26.08)$ & -29.73 & $<0.01$ \\
\hline Senses; taste \& smell & $23.60(28.89)$ & $64.66(31.66)$ & -41.05 & $<0.01$ \\
\hline Swallowing & $22.53(23.76)$ & $50.70(28.30)$ & -28.18 & $<0.01$ \\
\hline Problems with speech & $34.58(25.40)$ & $41.14(25.95)$ & -6.55 & $<0.01$ \\
\hline Social/feeding problems & $18.55(23.40)$ & $39.53(27.18)$ & -20.97 & $<0.01$ \\
\hline Interaction difficulties & $20.63(22.71)$ & $28.83(23.48)$ & -8.19 & $<0.01$ \\
\hline Sexuality & $37.69(32.90)$ & $47.12(35.25)$ & -9.43 & $<0.05$ \\
\hline Problems with teeth & $27.5(39.50)$ & 33.3 (42.9) & 5.8 & 0.682 \\
\hline Difficulty opening mouth & $23.99(35.50)$ & 41.98 (36.17) & -18.04 & $<0.01$ \\
\hline Dry mouth & $28.99(28.16)$ & 62.98 (33.09) & -33.98 & $<0.01$ \\
\hline Sticky Saliva & $29.92(29.79)$ & $64.72(32.11)$ & -34.79 & $<0.01$ \\
\hline Cough & $8.80(15.6)$ & $36.2(35.1)$ & -26.4 & $<0.01$ \\
\hline Feels Sick & $25.32(28.80)$ & $41.58(32.48)$ & -16.26 & $<0.01$ \\
\hline Takes analgesics & $48.77(50.11)$ & $19.21(39.49)$ & 29.55 & $<0.01$ \\
\hline Food supplement & $5.90(24.10)$ & $23.50(43.70)$ & -17.6 & 0.165 \\
\hline Feeding with a tube & $5.90(24.30)$ & $11.80(32.20)$ & -5.9 & 0.779 \\
\hline Weight loss & $62.0(49.11)$ & 21.51 (39.73) & 40.48 & $<0.01$ \\
\hline Weight gain & $22.50(43.70)$ & $46.10(51.40) 78$ & -23.60 & 0.202 \\
\hline
\end{tabular}


differentiated QOL of patients at the end of treatment; worse physical functioning and damage to the senses of taste and smell were reported by those in stages III and IV of the disease. Localization of the tumor at the end of treatment differentiated QOL in just two parameters. As for tumors located in the larynx and hypopharynx, those patients suffered from higher percentage of speech disorders that considerably decreased the QOL. However, patients who had the tumors in the oral cavity suffered from difficulty in opening their mouths thus negatively affecting the QOL. The patients treated with postoperative CRT demonstrated statistically significant decrease in body weight, impaired functioning in their life roles and constipation which had a negative impact on their QOL (Table 4).

\section{Discussion}

The demographic aspects of our subset of patients match the epidemiological profile of head and neck cancer patients reported in the literature, men over 45 years of age, exposed to one risk factor at least, such as exposure to sunlight, smoking or alcohol [10]. $44.5 \%$ of our patients were eligible for radiotherapy only while $33.3 \%$ were treated with radiotherapy plus chemotherapy. With the technological advances in radiotherapy techniques as well as the new chemotherapy protocols, there still remain the risks of increasing toxicity induced by chemoradiation with varying degrees of side effects, which may even cause treatment interruption, thus compromising

Table 4. The effect of clinical and social factors on QOL in 87 patients.

\begin{tabular}{|c|c|c|c|}
\hline Variables & Mean & Standard Deviation & p-Value \\
\hline \multicolumn{4}{|c|}{ Pain } \\
\hline Gender: Female & 60.52 & 24.97 & \\
\hline Male & 49.23 & 25.86 & $<0.05$ \\
\hline \multicolumn{4}{|c|}{ Senses } \\
\hline Age (years) $<60$ & 59.32 & 31.54 & \multirow{2}{*}{$<0.05$} \\
\hline$>60$ & 70.73 & 30.76 & \\
\hline Clincal Stage: I \& II & 46.08 & 37.97 & \multirow{2}{*}{$<0.05$} \\
\hline III \& IV & 65.25 & 30.65 & \\
\hline \multicolumn{4}{|c|}{ Life Role Performance } \\
\hline Smoking: Mod-Heavy & 69.35 & 27.11 & \multirow{2}{*}{$<0.01$} \\
\hline Never & 88.89 & 15.12 & \\
\hline \multicolumn{4}{|c|}{ Physical Performance } \\
\hline Clinical Stage: I \& II & 84.71 & 14.10 & \multirow{2}{*}{$<0.05$} \\
\hline III \& IV & 74.26 & 18.36 & \\
\hline \multicolumn{4}{|c|}{ Speech } \\
\hline Tumor Site: Lx \& hypophx & 45.34 & 24.87 & \multirow{2}{*}{$<0.05$} \\
\hline Oral Cavity & 31.94 & 24.24 & \\
\hline \multicolumn{4}{|c|}{ Mouth Opening } \\
\hline Lx \& Hypophx & 31.25 & 32.04 & \multirow{2}{*}{$<0.01$} \\
\hline Oral Cavity & 44.79 & 37.49 & \\
\hline \multicolumn{4}{|c|}{ Body Weight Decrease } \\
\hline ttt Method: CRT & 29.63 & 45.87 & \multirow{2}{*}{$<0.01$} \\
\hline RT & 6.98 & 25.78 & \\
\hline
\end{tabular}


the prognosis of the patient [11] [12].

In the questionnaire QLQ-C30 9 of the 30 analyzed components were worsened by treatment, and thus resulted in very significant negative impact on QOL. By the end of treatment all symptomatic scales, except diarrhea, achieved a maximum value. As for the functional scales they produced the lowest results, meaning a bad performance. Bjordal et al. analyzed 280 patients and reported very similar results [13]. According to those authors higher negative impact on the QOL occurred during the first and second month from the start of RT.

Bansal et al. [12], studied 45 head and neck patients treated with radiotherapy, they reported a relation between worsening of the physical function and an increase in fatigue, pain and loss of appetite. Blanco et al. [14] showed an increase in pain, fatigue and weight loss and decline on the functional scale, with loss of physical, social and emotional functions and role performance. Moreover, Scharloo et al. [15] studied 177 patients prospectively and reported an improvement in the emotional function alongside a worse social functioning throughout the follow up period.

In our patients, the highest reported negative effects were related to the loss of appetite. Changes were measured as the delta = difference between means; $(\Delta=39)$, losing the sense of taste and the increasing swallowing difficulties, first to solid food then semisolids and fluids, and increasing pain in the mouth and throat, thus causing the patients to lose their appetite and eventually show no desire to eat.

Additionally, three more components significantly influenced the QOL in our patients negatively; nausea and vomiting $(\Delta=18)$, pain $(\Delta=16)$ and fatigue $(\Delta=15)$. Notably the chemotherapy agents administered were the main cause of nausea and vomiting in the patients treated with CRT. Whereas RT was the cause of pain and the high value of delta points out the need for more effective pain medications in patients during radiation therapy or chemo-radiation therapy. The severity of pain was associated with tumor location and the method of RT. The largest magnitude of pain occuring between the beginning and the end of RT was reported during CRT in the laryngeal and hypopharyngeal tumors. In patients who had received CRT, the pain also increased in the same pattern. The delta for patients who reported fatigue at the end of treatment was $(\Delta=15)$, meaning that fatigue greatly and significantly worsens QOL. Notably, Women experienced greater fatigue than men. These findings are in concordance with those published by Wan et al, who analyzed 640 Head and Neck patients quality of life after treatment using the same EORTC questionnaires [16].

Our results showed that social functioning $(\Delta=9)$, life roles' functioning $(\Delta=8)$, and physical functions $(\Delta=$ 6) amounted to statistical significance. A result that is expected with RT lasting about 2 months where the patient's social activity almost stops and his/her physical condition declines markedly.

Our present study in the questionnaire QLQ-H\&N35 10 of the assessed factors proved to be of clinical significance $(\Delta \geq 10)$, definite increase in dry mouth, more viscid saliva with a significant affection of swallowing (p $<0.01$ ). Those changes may be partly accounted for by the surgical procedures utilized in the treatment of head and neck tumors. Other justifiable factors are the higher propensity of primary tumors in aerodigestive sites, such as the tongue and pharynx (22.7\% each), and non-surgical treatment, such as chemo-radiotherapy, in organ preservation protocols [17].

In our study, pain has declined by the end of treatment. The high standard deviation from the mean suggests a large variability in its perception. A logical explanation for the increase in the pain during treatment would be the opportunistic lesions arising from the radiotherapy and/or the chemotherapy, which is a call to increase the analgesic medication use to control it. Pain is common in patients with head and neck cancer. Epstein et al have reported that about half of their patients had pain before the start of any treatment, $81 \%$ of them during treatment, $70 \%$ at the end and only $36 \%$ continued to have pain six months or more after treatment. [18]

Upon analyzing effect of various factors on QOL results showed that the damaging of the sense of taste and smell by the end of treatment was reported by more patients in clinically stages III and IV and patients.

\section{Conclusion \& Recommendation}

Treatment modalities significantly affected the QOL in patients with HNSCC negatively. The location of the tumor, clinical stage, treatment modality, gender, age and habit of smoking had a statistically significant impact on QOL by the end of the treatment course. The greatest negative impact noted was severe or complete affection of the sense of taste and smell, weight loss, dry mouth, vicid saliva retention, pain, loss of appetite, nausea and vomiting as well as fatigue. Analyzing the selected factors documented that the tumor being in the larynx and/or hypopharynx they were associated with the severest impact on QOL. Assessing the quality of life of cancer pa- 
tients was complex, yet remained to be an essential tool and parameter to support recommended changes to attain a more effective support care protocol for the HNSCC patients [19]-[22].

\section{References}

[1] Murphy, B.A., Ridner, S., Wells, N. and Dietrich, M. (2007) Quality of Life Research in Head and Neck Cancer: A Review of the Current State of the Science. Critical Reviews in Oncology/Hematology, 62, 251-267. http://dx.doi.org/10.1016/j.critrevonc.2006.07.005

[2] Trask, P., Hsu, M. and McQuellon, R. (2009) Other Paradigms: Health-Related Quality of Life as a Measure in Cancer Treatment: Its Importance and Relevance. The Cancer Journal, 15, 435. http://dx.doi.org/10.1097/PPO.0b013e3181b9c5b9

[3] List, M.A. and Stracks, J. (2003) Quality of Life and Late Toxicities in Head and Neck Cancer. In: Brockstein, B.E. and Master, G.A., Eds., Head and Neck Cancer, Kluwer Academic Publishers, Norwell.

[4] Aaronson, N.K., Ahmedzai, S., Bergman, B., et al. (1993) The European Organization for Research and Treatment of Cancer QLQ-C30: A Quality-of-Life Instrument for Use in International Clinical Trials in Oncology. Journal of the National Cancer Institute, 85, 365-376. http://dx.doi.org/10.1093/jnci/85.5.365

[5] Bjordal, K. and Kaasa, S. (1992) Psychometric Validation of the EORTC Core Quality of Life Questionnaire, 30-Item Version and a Diagnosis-Specific Module for Head and Neck Cancer Patients. Acta Oncologica, 31, 311-321. http://dx.doi.org/10.3109/02841869209108178

[6] Bjordal, K., Hammerlid, E., Ahlner-Elmqvist, M., et al. (1999) Quality of Life Head and Neck Cancer Patients: Validation of the European Organization for Research and Treatment of Cancer Quality of Life Questionnaire-H\&N35. Journal of Clinical Oncology, 17, 1008-1019.

[7] Sobin, L.H. and Wittekind, C. (2002) TNM Classification of Malignant Tumors. 6th Edition, Wiley-Liss, New York, 264.

[8] King, M.T. (1996) The Interpretation of Scores from the EORTC Quality of Life Questionnaire QLQ-C30. Quality of Life Research, 5, 555-567. http://dx.doi.org/10.1007/BF00439229

[9] Osoba, D., Rodrigues, G., Myles, J., Zee, B. and Pater, J. (1998) Interpreting the Significance of Changes in HealthRelated Quality of Life Scores. Journal of Clinical Oncology, 16, 139-144.

[10] American Cancer Society (2006) Cancer Facts \& Figures 2006. American Cancer Society, Atlanta.

[11] Braam, M.P., Roesink, J.M., Raaijmakers, C.P., Busschers, W.B. and Terhaard, C.H. (2007) Quality of Life and Salivary Output in Patients with Head-and-Neck Cancer Five Years after Radiotherapy. Radiation Oncology, $2,3$. http://dx.doi.org/10.1186/1748-717X-2-3

[12] Bansal, M., Mohanti, B.K., Shah, N., Chaudhry, R., Bahadur, R. and Shukla, N.K. (2004) Radiation Related Morbidities and Their Impact on Quality of Life in Head and Neck Cancer Patients Receiving Radical Radiotherapy. Quality of Life Research, 13, 481-488. http://dx.doi.org/10.1023/B:QURE.0000018491.80646.bc

[13] Bjordal, K., Ahlner-Elmqvist, M., Hammerlid, E., Boysen, M., Evensen, J.F., Biörklund, A., Jannert, M., Westin, T. and Kaasa, S. (2001) A Prospective Study of Quality of Life in Head and Neck Cancer Patients. Part II: Longitudinal Data. Laryngoscope, 111, 1440-1452. http://dx.doi.org/10.1097/00005537-200108000-00022

[14] Blanco, M.A.-B. and González-Botas, J.H. (2011) Quality of Life Evolution in Patients after Surgical Treatment of Laryngeal, Hypopharyngeal or Oropharyngeal Carcinoma. Acta Otorrinolaringologica (English Edition), 62, 103-112. http://dx.doi.org/10.1016/S2173-5735(11)70019-9

[15] Scharloo, M., Baatenburg de Jong, R.J., Langeveld, T.P., van Velzen-Verkaik, E., Doorn-Op den Akker, M.M. and Kaptein, A.A. (2010) Illness Cognitions in Head and Neck Squamous Cell Carcinoma: Predicting Quality of Life Outcome. Supportive Care in Cancer, 18, 1137-1145. http://dx.doi.org/10.1007/s00520-009-0728-X

[16] Wan Leung, S., Lee, T.F., Chien, C.Y., Chao, P.J., Tsai, W.L. and Fang, F.M. (2011) Health-Related Quality of Life in 640 Head and Neck Cancer Survivors after Radiotherapy Using EORTC QLQ-C30 and QLQ-H\&N35 Questionnaires. BMC Cancer, 11, 128. http://dx.doi.org/10.1186/1471-2407-11-128

[17] Martino, R. and Ringash, J. (2008) Evaluation of Quality of Life and Organ Function in Head and Neck Squamous Cell Carcinoma. Hematology/Oncology Clinics of North America, 22, 1239-1256. http://dx.doi.org/10.1016/j.hoc.2008.08.011

[18] Epstein, J.B., Hong, C., Logan, R.M., Barasch, A., Gordon, S.M., Oberle-Edwards, L., et al. (2010) A Systematic Review of Orofacial Pain in Patients Receiving Cancer Therapy. Supportive Care in Cancer, 18, 1023-1031. http://dx.doi.org/10.1007/s00520-010-0897-7

[19] Petruson, K.M., Silander, E.M. and Hammerlid, E.B. (2005) Quality of Life as Predictor of Weight Loss in Patients with Head and Neck Cancer. Head \& Neck, 27, 302-310. http://dx.doi.org/10.1002/hed.20172 
[20] Infante-Cossio, P., Torres-Carranza, E., Cayuela, A., Gutierrez-Perez, J.L. and Gili-Miner, M. (2009) Quality of Life in Patients with Oral and Oropharyngeal Cancer. International Journal of Oral and Maxillofacial Surgery, 38, $250-255$. http://dx.doi.org/10.1016/j.ijom.2008.12.001

[21] Hammerlid, E., Bjordal, K. and Ahler-Elmqvist, M. (2001) A Prospective Study of Quality of Life in Head and Neck Cancer Patients. Part I: At Diagnosis. Laryngoscope, 111, 669-680. http://dx.doi.org/10.1097/00005537-200104000-00021

[22] Spych, M., Gottwald, L., Klonowicz, M., Biegała, M., Bibik, R. and Fijuth, J. (2010) Clinical Research? The Analysis of Prognostic Factors Affecting Post-Radiation Acute Reaction after Conformal Radiotherapy for Non-Small Cell Lung Cancer. Archives of Medical Science, 6, 756-763. http://dx.doi.org/10.5114/aoms.2010.17092 